Distinctive patterns of macrolides, lincosamides, and streptogramins resistance phenotypes and determinants among Staphylococcus aureus populations in Hong Kong

Pak-Leung Ho*, Eileen L. Lai, Maggie Y. Chan, Kin-Hung Chow

Department of Microbiology and Carol Yu Centre for Infection, The University of Hong

Kong, Hong Kong SAR, CHINA

\title{
Correspondance to
}

Pak-Leung Ho, MD, FRCPath

plho@hkucc.hku.hk 
Sir,

In staphylococci, resistance to macrolides, lincosamides, and streptogramin B antibiotics (MLS phenotype) is caused by a ribosomal methylase encoded by the erm genes. Expression of resistance to clindamycin can be either inducible or constitutive. The inducible phenotype is expressed only in the presence of macrolides, and these strains appear to be susceptible to clindamycin in vitro. However, reports of treatment failures have led to concerns about the efficacy of clindamycin for strains with inducible (iMLS) phenotypes [1], especially when oral clindamycin may be used for outpatient treatment of infections caused by community-associated methicillin-resistant Staphylococcus aureus (CA-MRSA). In Hong Kong, studies have showed that multiple lineages of CA-MRSA are emerging [2]. Here, we determine the prevalence of MLS phenotypes and their resistance determinants among our CA-MRSA isolates and compared them with methicillin-sensitive S. aureus (MSSA) and healthcare-associated (HA)-MRSA.

Susceptibility of the isolates to erythromycin and clindamycin were determined by the disc diffusion method. The D-test was used to detect inducible resistance to clindamycin. Quality control strains were included on each day of testing. The relatedness of the strains were characterized by spa typing and/or multilocus sequencing. MRSA strains were additionally tested by SCCmec multiplex PCR [2,3]. Multiplex PCR assays for the erm A, ermB, ermC, mef and msrA genes were performed using previously described primers [4]. 
A total of 422 S. aureus isolates including 185 MSSA group, 107 HA-MRSA and 130

CA-MRSA isolates collected in Hong Kong during 2004-2007 from diverse sources were tested [2,3]. Overall, the erythromycin resistance rate was 36.7\% (155/422) including 60.7\% (65/107) for HA-MRSA, 26.9\% (35/130) for CA-MRSA and 29.7\% (55/185) for MSSA. Among erythromycin-resistant (ery-R) isolates, 24.5\% (38/155) and 67.1\% (104/155) had constitutive (cMLS phenotype) and inducible (iMLS phenotype) resistance to clindamycin, respectively. The remaining 13 erythromycin-resistant isolates (11 CA-MRSA and 2 MSSA) had the M phenotype (i.e. resistant to erythromycin only). Another four isolates (all CAMRSA) were resistant to clindamycin alone (L phenotype). The iMLS phenotype predominated among MSSA (78.1\%, 43/55) and HA-MRSA (93.8\%, 61/65), while the cMLS phenotype was prevalent among CA-MRSA (68.6\%, 24/35).

The 155 ery-R isolates were tested for the presence of MLS resistance genes (Table 1). At least one erm gene was detected in 98.6\% (140/142) of the isolates with cMLS or iMLS phenotype. Among ery-R MSSA isolates, ermA gene (67.3\%, 37/55) predominated, followed by $\operatorname{erm} C(18.2 \%, 10 / 55)$ and ermB $(10.9 \%, 6 / 55)$. By comparison, $56.9 \%$ and $44.6 \%$ of the ery-R HA-MRSA were found to possess ermC and ermA, respectively. In CA-MRSA, the MLS phenotype was largely attributed to ermB and the M phenotype to msrA genes.

In MRSA, the MLS determinants were largely distributed among clonally related strains. Thirty-one (86.1\%) of the 36 ermC positive HA-MRSA isolates were of the 
CC45/SCCmec IV/V clone (t1081 or related spa types). The remaining five ermC HA-MRSA isolates belonged to CC7/SCCmec V lineage ( $n=2, t 1081)$, EMRSA-15 ( $n=2$, ST22, t032) and ST188 (n=1, t198). Similarly, 86.2\% (25/29) of the ermA positive and the singleton ermA + ermC positive HA-MRSA isolates were of the CC5/SCCmec II clone (t002). For CAMRSA, all of the $20 \mathrm{ermB}$ positive and two ermB + ermC positive isolates were of the CC59/SCCmec V clone. All the 11 msrA positive CA-MRSA isolates belonged to the ST8/SCCmec IV clone (i.e. USA300) and they have spa types t008 $(n=9)$ or t2196 $(n=2)$. Conversely, the MSSA strains with ermA, ermB or ermC genes were genetically diverse. Analysis of 28 ery-R MSSA isolates showed that they fell into 17 different spa types. Types with $>1$ isolates included t338 $(n=7)$, t437 $(n=3)$, t002 $(n=2)$, t091 (n=2) and t127 (n=2). The following spa types had one isolate each: t012, t034, t1244, t1399, t2421, t337, t3485, t363, t732, t899 and t930.

This study revealed distinctive patterns of erm and msrA genes among S. aureus populations. The majority of ery-R MSSA and HA-MRSA had the iMLS phenotype encoded by either the ermA or ermC genes. In contrast, ery-R CA-MRSA isolates often had the cMLS phenotype and ermB. The findings indicate both independent acquisitions of the erm genes as well as spread of specific resistant clones. As observed previously, ermA is usually inducible while ermB is expressed constitutively, and the expression of ermC may be either inducible or constitutive [5]. A small proportion of the ermA positive strains had the cMLS phenotype. 
Such change from inducible to constitutive expression could possibly be due to gene duplication, deletion or point mutation in the erm regulatory regions [4]. With the exception of the CA-MRSA isolates related to the USA300 clone, the $m s r A$ was rare among the other $S$. aureus populations; suggesting that the gene may have been recently introduced to our community. In conclusion, our findings demonstrate that emergence of CA-MRSA in our locality was associated with emergence of the ermB and msrA genes that were uncommon among the MSSA and HA-MRSA populations.

Funding: The work was supported by research grants from the Research Fund for the Control of Infectious Diseases (RFCID) of the Health and Food Bureau of the Hong Kong SAR Government.

Competing interests: None declared.

Ethical approval: not required. 


\section{Table 1}

Prevalence of macrolide resistance genes among 155 erythromycin-resistant Staphylococcus aureus isolates, 2004-2007

$$
\text { No (\% by S. aureus group) }
$$

\begin{tabular}{|c|c|c|c|c|c|}
\hline & ermA & ermB & ermC & $m s r A$ & none \\
\hline \multicolumn{6}{|c|}{ MSSA (n=55) } \\
\hline cMLS & 0 & $6(10.9)$ & $4(7.3)$ & & \\
\hline iMLS & 37 (67.3) & 0 & $6(10.9)$ & & \\
\hline M & 0 & 0 & 0 & 1 & 1 \\
\hline \multicolumn{6}{|c|}{ HA-MRSA $(n=65)$} \\
\hline cMLS & $2(3.1)$ & 0 & $2(3.1)$ & & \\
\hline iMLS & 27 (41.5) & 0 & $35(53.8)^{\mathrm{a}}$ & & \\
\hline \multicolumn{6}{|c|}{ CA-MRSA $(n=35)$} \\
\hline cMLS & 0 & $22(62.9)$ & $2(5.7)^{b}$ & & 2 \\
\hline M & 0 & 0 & 0 & 11 & \\
\hline
\end{tabular}

${ }^{\text {a }}$ One isolate positive for both ermA and ermC genes.

b Two isolates positive for both ermB and ermC 


\section{References}

[1] Lewis JS, Jorgensen JH. Inducible clindamycin resistance in Staphylococci: should clinicians and microbiologists be concerned? Clin Infect Dis 2005; 40:280-5.

[2] Ho PL, Cheung C, Mak GC et al. Molecular epidemiology and household transmission of community-associated methicillin-resistant Staphylococcus aureus in Hong Kong. Diagn Microbiol Infect Dis 2007; 57:145-51.

[3] Ho PL, Lai EL, Chow KH, Chow LS, Yuen KY, Yung RW. Molecular epidemiology of methicillin-resistant Staphylococcus aureus in residential care homes for the elderly in Hong Kong. Diagn Microbiol Infect Dis 2008; 61:135-42.

[4] Roberts MC. Update on macrolide-lincosamide-streptogramin, ketolide, and oxazolidinone resistance genes. FEMS Microbiol Lett 2008; 282:147-59.

[5] Eady EA, Ross JI, Tipper JL, Walters CE, Cove JH, Noble WC. Distribution of genes encoding erythromycin ribosomal methylases and an erythromycin efflux pump in epidemiologically distinct groups of staphylococci. J Antimicrob Chemother 1993; 31:211-7. 